\title{
The use of multivariate cross prediction methods in the breeding of a clonally reproduced crop (Solanum tuberosum)
}

\author{
J. Brown and \\ P. D. S. Caligari*
}

Scottish Crop Research Institute, Pentlandfield, Roslin, Midlothian EH25 9RF, Scotland.

Three methods of cross prediction were examined namely multivariate probabilities, the sum of ranks and the frequency of genotypes in a sample that transgress set target values. The characters tuber weight, mean tuber weight, number of tubers and regularity of tuber shape were examined. It was found that a sample as small as 25 clones provided good predictions (as judged by the observed frequencies in larger progeny samples examined in various environments). The best estimates were obtained using multivariate probabilities based on the means, within progeny variances and the phenotypic correlation between variates. The rankings of the crosses according to these multivariate probabilities provided good indications of the number of clones which survived selection in an actual breeding scheme. Thus it is suggested that an empirical examination of a sub-sample of the progeny from a cross could be used to determine the crosses which would have the highest probability of producing new commercial potato cultivars with improved characteristics.

\section{INTRODUCTION}

Univariate cross prediction has been applied to a number of inbreeding species based on the work of Jinks and Pooni (1976) with Nicotiana rustica. Predictions of the proportion of recombinant inbred lines that will transgress either, or both, parents are based on the evaluation of the integral:

$$
\int_{T}^{\infty} f\left(x_{i}\right) d x_{i}
$$

where the variate of interest is normally distributed, and the function $f\left(x_{i}\right)$ is based on $m$, the mean of all possible inbreds for the character and $D$, the additive genetic variance for the character. The prediction parameters can be estimated by using various, appropriate experimental methods such as the triple test cross (Jinks and Pooni, 1976 and Snape, 1982), evaluation of $F_{\infty}$ lines produced through doubled haploid or single seed descent (Caligari, Powell and Jinks, 1985) or raising $\mathrm{F}_{3}$ 's (Jinks and Pooni, 1980; Snape and Parker, 1986; Thomas, Tapsell and Powell, 1986).

* Present address: Department of Agricultural Botany, Plant Science Laboratories, The University of Reading, Whiteknights, P.O. Box 221, Reading RG6 2AS, U.K.
After the initial cross the breeding of a clonally reproduced crop, such as potatoes (Solanum tuberosum), is not affected by genetic segregation as would be an inbreeding, sexually reproducing species, as for example barley or wheat (Caligari and Brown, 1986). Nevertheless it has been shown that early generation clonal selection in potato breeding is generally ineffective (Caligari, Brown and Abbott, 1986; Brown, Caligari, Mackay and Swan, 1987). This lead Caligari and Brown (1986) to examine univariate cross prediction methods in potato breeding. They found that predictions based on breeders' preference scores (a visual assessment of commercial worth on a 1-9 scale of increasing desirability), estimated in any of the early clonal generations, provided a good indication of the potential worth of the cross, in later generations.

A new cultivar will usually not be successful simply because of high expression in a single character, but rather it needs to have an overall improvement in a number of morphological, pathological or quality characters. Caligari and Brown (1986) partially overcome this problem by considering a variate, breeders' preference, which was based on a visual assessment of a number of characters by the breeder. This has limitations since other characters, such as disease resistance 
or cooking quality cannot readily be incorporated into this one variate. Thus more than one character must be considered. Evaluation of crosses in a breeding programme for more than a single character has been shown to be effective in Nicotiana rustica (Pooni and Jinks, 1978) and also in Hordeum vulgare (Powell, Caligari, McNicol and Jinks, 1985; Thomas, Tapsell and Powell, 1986). Such multivariate predictions were based on the estimation of the multi-normal distribution functions with parameters $m$, the mean of all possible inbred lines of each character, $D$, the additive genetic variance of each character and $r_{D}$ the additive genetic correlation between the characters of interest.

This paper examines the feasibility of using similar multivariate cross prediction methods in a potato breeding programme. Predicted frequencies of clones were estimated (based on a sample of genotypes from each cross) which would exceed set target values for total tuber weight, mean tuber weight, number of tubers per plant and regularity of tuber shape. These predictions can be compared with the frequencies observed when examining a large number of clones from each cross. Since in $S$. tuberosum the predictions are over clonal rather than sexual generations, the above estimates involving the additive effects can be replaced simply by estimates of the genetic effects and in practice the phenotypic effects were used in this study, as described by Caligari and Brown (1986).

\section{MATERIALS AND METHODS}

The progeny from eight potato crosses (C1-C8) were examined. The growing conditions and experimental design used for the seedlings in the glasshouse, and the clones in the first clonal year were described by Brown, Caligari, Mackay and Swan (1984). Those for the second and third clonal generations were given by Brown et al., (1987). The details are thus only given briefly here.

In 1981, 200 seedlings from each cross were grown in $10 \mathrm{~cm}$ square pots in an aphid proof glasshouse. The following year (1982) every clone which produced sufficient tubers at the seedling stage was planted in the field at two locations, Blythbank (BB) and Murrays (MURR) Farms. Where sufficient tubers were available each clone was grown as a single-plant plot in two completely randomised blocks at each location. In 1983, 70 clones were taken at random from each cross and grown at BB and MURR. Each clone was represented by two, three-plant, plots in each of two completely randomised blocks at each location. The following year the number of clones per cross was further reduced at random to 25 . These were grown in trials similar to those in 1983 but the plot size was increased to five plants.

$B B$ is the farm used by the Potato Breeding Department at the Scottish Crop Research Institute (SCRI) for the production of healthy seed tubers. These are subsequently used to plant assessment trials at other locations. It is however the only site used for clonal selection of the first and second clonal years of the breeding scheme used at SCRI. MURR is used for yield and other ware assessment trials. To avoid the risk of virus infection, transmitted by aphids, the BB site is planted later and harvested earlier than the MURR site. Hence BB has a shorter growing season than MURR. Tubers from the BB trial were always used as the seed source for the following year's trials at both sites.

In each year, at each site, individual plots were harvested and taken into storage. The total weight and number of tubers were recorded for each plot and from these, mean tuber weights were calculated. Regularity of tuber shape was visually assessed on a 1-9 scale of increasing regularity (i.e., $1=$ very irregular to $9=$ very regular, smooth shape). The total tuber yield and number of tubers were converted to a per-plant basis before being analysed. One of the variates, number of tubers, showed a significantly skewed distribution but was found to conform to normality after being transformed, by taking the square root.

Three methods of cross prediction were examined:

(1) the multivariate probability

(2) The sum of ranks.

(3) Frequency in a sub-sample.

Multivariate probabilities were calculated using a computer program developed at SCRI (Powell et al., 1985) which was based on an algorithm by Schervish (1984). The parameters used in the probability estimates for each cross were the mean and phenotypic variance for each of the characters (total tuber weight, mean tuber weight, number of tubers and regularity of shape) together with the phenotypic correlations between them. The sum of ranks (for a detailed description see Kendel, 1962) was obtained by ranking the eight crosses for each of the four individual variates then summing them to give a total for each. The observed frequencies in the sub-samples as well as over all clones were obtained by simply counting the number of clones that we:e observed to be greater than the target values set for each of the four variates simultaneously. The target values used for each year and site were the overall means of each variate, averaged over all eight crosses. 
Table 1 Coefficients obtained by correlating the observed frequency of clones (based on all the clones examined) with the predicted frequency of phenotypes greater than the mean value for all four characters (total tuber weight, mean tuber weight, number of tubers per plant and uniformity of tuber shape) within each of five different environments. The predicted frequencies are based on (1) multivariate probabilities; (2) sum of ranks and (3) the observed frequency in a sub-sample of the progeny

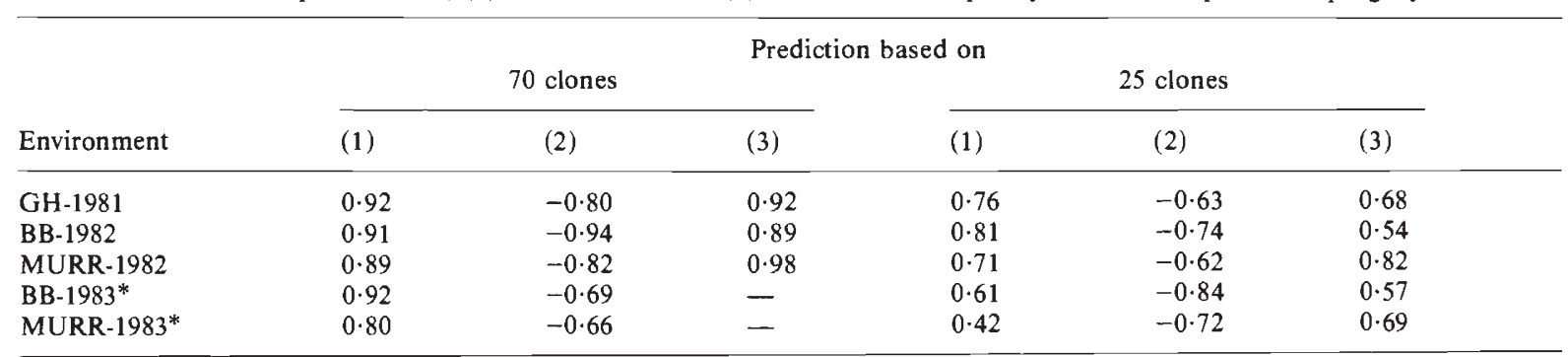

* Observed frequencies based on 70 clones per cross, all others being based on 200 clones.

\section{RESULTS}

The effectiveness of estimation within a single environment will be considered first. Two sample sizes, 70 clones per cross and 25 clones per cross were examined. The predictions based on both sub-sample sizes were correlated with the frequency of clones that were observed when all clones were examined (table 1). It should be noted that the correlation coefficient with the sum of ranks will tend to be negative because the lower the sum the greater is the probability of the cross producing desirable clones. As expected the predictions using a sub-sample of 70 clones per cross gave higher correlation coefficients with the observed frequencies than the 25 clone sample. The multivariate probability predictions, in general, resulted in higher correlation coefficients than either the sum of ranks or observed sub-sample frequencies based on 70 clones per cross. The sum of ranks produced higher correlation coefficients than the sub-sample frequency with a 25 clone sub-sample. However, the reverse was found when a larger sub-sample (70 clones per cross) was examined. There was a loss of accuracy in estimating the multivariate probabilities or sum of ranks when the sub-sample examined was reduced from 70 to 25 clones per cross but it was not large. The observed sub-sample frequencies showed much lower correlations when the number of clones was reduced. Overall however, it was found that a 25 clone sub-sample per cross produced a reasonable estimate of the observed frequency of clones within the same environment.

The target values used to define the lower bounds of total tuber weight, mean tuber weight, number of tubers and regularity of tuber shape in the predictions were the mean value of each character, averaged over all crosses, in each environment. The frequency of clones observed to be greater than the target value for the four variates simultaneously in a sample of 200 clones per cross and from a 25 clone per cross sample were estimated (table 2). The predicted frequencies, based on multivariate probabilities and the sum of rankings based on the same target values for the four variates are also shown in table 2 .

Cross prediction in most practical plant breeding situations will involve the estimation of the worth of progeny in one environment, by evaluation of a sub-sample of genotypes from each cross under investigation. The prediction is to provide an estimate of the performance of the cross in the later stages of a selection scheme when larger number of clones are grown from each cross. This was simulated by correlating predicted frequencies (based on evaluation of 25 clones per cross) with the observed frequencies based on a larger sample of genotypes in the different years and sites. The coefficients from correlating the observed frequencies with those obtained from the predictions using multivariate probabilities, sum of ranks and simply observed in the sub-sample were generally significantly different from zero despite being based on only eight crosses (table 3 ). As with the comparison between predicted and observed in a single environment, the multivariate probability predictions provided the best estimate.

Both the multivariate probability and observed frequency based on 25 clone sub-samples estimated on seedlings in the glasshouse resulted in an extremely low coefficient when correlated with the observed frequency from $\mathrm{BB}$ in the second clonal year. This result mainly traced to one of the eight crosses. This cross showed the lowest number of tubers per seedling in the glasshouse but had a very high number of tubers at $\mathrm{BB}$ in the second clonal year (1983). The reason for this is not apparent. 
Table 2 Observed frequency of clones ( 200 and 70 clones per sample), expected frequency based on a 25 clone per cross sample using multivariate probability (MVP), frequency of clones in the sub-sample and the sum of the ranks. All predictions were for clones scoring greater than the overall mean of all crosses in the glasshouse (GH) 1981 and at Blythbank (BB) and Murrays (MURR) in 1982 and 1983

\begin{tabular}{|c|c|c|c|c|c|c|}
\hline & \multirow[b]{2}{*}{ Cross } & \multirow{2}{*}{$\begin{array}{l}1981 \\
\mathrm{GH}\end{array}$} & \multicolumn{2}{|l|}{1982} & \multicolumn{2}{|l|}{1983} \\
\hline & & & BB & MURR & $\mathrm{BB}$ & MURR \\
\hline Observed & $\begin{array}{l}\mathrm{C} 1 \\
\mathrm{C} 2 \\
\mathrm{C} 3 \\
\mathrm{C} 4 \\
\mathrm{C} 5 \\
\mathrm{C} 6 \\
\mathrm{C} 7 \\
\mathrm{C} 8\end{array}$ & $\begin{array}{r}6 \cdot 06 \\
12 \cdot 42 \\
15 \cdot 03 \\
8 \cdot 57 \\
0 \cdot 54 \\
4 \cdot 35 \\
15 \cdot 51 \\
3 \cdot 00\end{array}$ & $\begin{array}{r}12 \cdot 50 \\
3 \cdot 17 \\
2 \cdot 78 \\
9 \cdot 77 \\
4 \cdot 71 \\
5 \cdot 55 \\
16 \cdot 57 \\
0.71\end{array}$ & $\begin{array}{r}12.21 \\
7.63 \\
6.80 \\
10.46 \\
8.38 \\
7.63 \\
30.91 \\
2.20\end{array}$ & $\begin{array}{r}28 \cdot 58 \\
7 \cdot 25 \\
2 \cdot 86 \\
15 \cdot 94 \\
14 \cdot 29 \\
14 \cdot 29 \\
10 \cdot 00 \\
5 \cdot 15\end{array}$ & $\begin{array}{r}11 \cdot 42 \\
10.00 \\
8.57 \\
5.71 \\
4.28 \\
12.58 \\
7.41 \\
0.00\end{array}$ \\
\hline MVP & $\begin{array}{l}\mathrm{C} 1 \\
\mathrm{C} 2 \\
\mathrm{C} 3 \\
\mathrm{C} 4 \\
\mathrm{C} 5 \\
\mathrm{C} 6 \\
\mathrm{C} 7 \\
\mathrm{C} 8\end{array}$ & $\begin{array}{r}8 \cdot 32 \\
13 \cdot 00 \\
5 \cdot 56 \\
8 \cdot 19 \\
0 \cdot 08 \\
7 \cdot 49 \\
15 \cdot 71 \\
2 \cdot 29\end{array}$ & $\begin{array}{r}10.32 \\
6.97 \\
0.55 \\
5.54 \\
4.81 \\
9.01 \\
11.64 \\
1.21\end{array}$ & $\begin{array}{r}18 \cdot 28 \\
9.65 \\
5 \cdot 37 \\
15 \cdot 39 \\
9 \cdot 10 \\
13 \cdot 23 \\
20.94 \\
0.00\end{array}$ & $\begin{array}{r}15 \cdot 17 \\
8.41 \\
4 \cdot 30 \\
11.06 \\
15 \cdot 79 \\
22.71 \\
13.98 \\
5.89\end{array}$ & $\begin{array}{r}12.98 \\
11.78 \\
3.41 \\
1.99 \\
15.08 \\
6.89 \\
7.62 \\
0.04\end{array}$ \\
\hline Observed in sub-sample & $\begin{array}{l}\mathrm{C} 1 \\
\mathrm{C} 2 \\
\mathrm{C} 3 \\
\mathrm{C} 4 \\
\mathrm{C} 5 \\
\mathrm{C} 6 \\
\mathrm{C} 7 \\
\mathrm{C} 8\end{array}$ & $\begin{array}{r}8 \cdot 00 \\
16 \cdot 67 \\
4 \cdot 17 \\
4 \cdot 17 \\
0.00 \\
4 \cdot 17 \\
12 \cdot 50 \\
0 \cdot 00\end{array}$ & $\begin{array}{l}8.00 \\
4.17 \\
0.00 \\
0.00 \\
0.00 \\
4.17 \\
4.17 \\
0.00\end{array}$ & $\begin{array}{r}23.64 \\
0.00 \\
0.00 \\
4.17 \\
4.17 \\
0.00 \\
26.09 \\
0.00\end{array}$ & $\begin{array}{r}16 \cdot 00 \\
8 \cdot 33 \\
4 \cdot 17 \\
8 \cdot 33 \\
12 \cdot 50 \\
20 \cdot 83 \\
18 \cdot 33 \\
4 \cdot 17\end{array}$ & $\begin{array}{r}11 \cdot 11 \\
5 \cdot 88 \\
5 \cdot 00 \\
0 \cdot 00 \\
5 \cdot 26 \\
5 \cdot 00 \\
6 \cdot 76 \\
0.00\end{array}$ \\
\hline Sum of ranks & $\begin{array}{l}\mathrm{C} 1 \\
\mathrm{C} 2 \\
\mathrm{C} 3 \\
\mathrm{C} 4 \\
\mathrm{C} 5 \\
\mathrm{C} 6 \\
\mathrm{C} 7 \\
\mathrm{C} 8\end{array}$ & $\begin{array}{l}12 \\
13 \\
20 \\
13 \\
27 \\
22 \\
13 \\
24\end{array}$ & $\begin{array}{l}15 \\
21 \\
24 \\
16 \\
22 \\
13 \\
13 \\
20\end{array}$ & $\begin{array}{l}18 \\
23 \\
19 \\
11 \\
20 \\
14 \\
12 \\
27\end{array}$ & $\begin{array}{r}7 \\
21 \\
29 \\
20 \\
17 \\
10 \\
15 \\
25\end{array}$ & $\begin{array}{l}16 \\
22 \\
14 \\
22 \\
18 \\
12 \\
14 \\
26\end{array}$ \\
\hline
\end{tabular}

\section{DISCUSSIONS AND CONCLUSIONS}

All three methods used to predict which crosses would produce the highest frequencies of clones, having expression greater than the targets for all four variates, in later clonal generations proved to be successful. However, cross prediction based on the evaluation of the multi-normal distribution proved to be the best. It was found that even when the cross means, within progeny variances and correlations between variates were estimated in the most aytpical environment, that of seedlings grown in a glasshouse, a good indication of the frequency of clones that would transgress the target values was obtained. The prediction based on the observed frequency of clones in a sub-sample gave the poorest estimate. The weakness of these latter estimates is almost certainly due to the effects that sampling variation can play when so few clones are involved. Inspection of table 1 , where a 70 clone sample was used, showed that correlations between such predicted frequencies and those observed were considerably larger. Thus supporting the contention of the overriding effects of sampling variation when using the observed frequency in a sub-sample as a prediction system unless the number of clones is reasonably large, at least greater than 25 .

It was interesting that the relative magnitude of the sum of ranks gave good estimates of the observed frequencies. This is perhaps not surprising as the target values set were in fact the mean value of each variate, averaged over all clones and crosses. Caligari and Brown (1985) found that using univariate prediction in potatoes the genotypic, or phenotypic, variance of each cross 
Table 3 Coefficients obtained by correlating observed frequency of clones (based on all clones per cross) against predicted frequency (based on 25 clones per cross) estimated by using (a) the multivariate probability integral, (b) the observed frequency in the sub-sample and (c) the sum of the ranks. All predictions are of the clones in a cross that are greater than the mean value for total, mean tuber weight, number of tubers per plant and regularity of tuber shape

Observed

GH-1981

a. 0.75

b. 0.69

c. -0.63

BB-1982

a. $0.62 \quad 0.80$

b. $0.40 \quad 0.54$

c. $-0.65 \quad-0.74$

MURR-1982

a. $0.71 \quad 0.71$

b. $0.50 \quad 0.38$

c. $-0.51 \quad-0.57 \quad-0.62$

0.79

0.82

BB-1983*

a. $0.03 \quad 0.61$

b. $0.01 \quad 0.63$

c. $-0.34-0.55$

0.64

0.56

$-0.39 \quad-0.85$

$0 \cdot 61$

0.57

MURR-1983*

$\begin{array}{lr}\text { a. } 0.51 & 0.59 \\ \text { b. } 0.53 & 0.72 \\ \text { c. }-0.46 & -0.38\end{array}$

0.56

$0 \cdot 22$

$-0.43$

0.49

0.58

$-0.55$

0.42

0.69

$-0.74$

GH-1981 BB-1982

MURR-1983 BB-1983

MURR-1983

Expected

* = Observed frequencies based on 70 clones per cross, all other observed frequencies are based on 200 clones per cross. Correlations in italics are observed and predicted in the same environment.

added little to the univariate prediction. They did, however, observe that the within progeny variance became of greater importance when the target value was increased. Perhaps the same would be true here. With the target value set as the mean of each variate then the cross' means (directly related to the ranking) are of great importance. If however the target values are increased, the variance of each variate and also the covariances between variates are likely to be increasingly important in the predictions. In these cases the sum of rankings would not provide such a good prediction as the ranks are only based on the mean value of each variate for each cross. With the use of electronic computers it is now possible to calculate easily and quickly areas under a multi-normal distribution (Powell, Caligari, McNichol and Jinks, 1985). The method of cross evaluation for more than a single character using the sum of the ranks could however be very useful where highly powerful computers are not available or where many crosses are to be examined. In any case in the context of a practical potato breeding programme these target values used here may well give acceptable results and therefore the sum of the ranks would remain a reasonable predictor.

Clones examined in this study were entered into the normal selection scheme of the Potato
Breeding Department at SCRI. Although the clones were a random sample, in that no conscious selection had been carried out, they were entered into the scheme at the third clonal year stage in 1984. After that time, these clones were treated in exactly the same way as the other breeding material and clones only survived to the next year's trial according to the normal selection criteria. The number of clones from each cross that were considered suitable for trial in the fourth, fifth, and sixth clonal year (1985 to 1987 inclusively) are shown in table 4 . Alongside these data are the cross rankings, according to the multivariate probability predictions estimated from seedlings and also from BB and MURR in the first clonal year. The first point to note is that in total, over all crosses in the breeding scheme, including material from sources other than the crosses reported here, there were only 39 selections made from the fifth clonal year to give the clones in the sixth year. Clones which derived from this experiment accounted for just under one quarter of the total. It should be remembered that these clones derived from a random sample of only 200 clones at the third clonal year stage while the other material was derived from a larger population $(40,000$ seedlings) which were represented by (973) at this stage. This suggests that either, despite efforts to the contrary, the eight 
Table 4 The rankings of crosses according to multivariate probability predictions estimated on seedlings in the glasshouse $(\mathrm{GH})$ and at BB and MURR in the first clonal year. Also given are the number of clones from each cross which were retained to the fourth, fifth and sixth clonal year of the commercial breeding scheme at SCRI

\begin{tabular}{|c|c|c|c|c|c|c|}
\hline \multirow[b]{2}{*}{ Cross } & \multicolumn{3}{|c|}{ Predicted rankings } & \multicolumn{3}{|c|}{$\begin{array}{l}\text { Number of clones } \\
\text { that survived to year }\end{array}$} \\
\hline & 1981 & 1982 & 1982 & Four & Five & Six \\
\hline $\mathrm{C} 1$ & 3 & 2 & 2 & 15 & 3 & 2 \\
\hline $\mathrm{C} 2$ & 2 & 4 & 5 & 9 & 3 & 2 \\
\hline $\mathrm{C} 3$ & 6 & 8 & 7 & 1 & 0 & 0 \\
\hline $\mathrm{C} 4$ & 4 & 5 & 3 & 2 & 0 & 0 \\
\hline $\mathrm{C} 5$ & 8 & 6 & 6 & 10 & 3 & 1 \\
\hline C6 & 5 & 3 & 4 & 11 & 6 & 1 \\
\hline C7 & 1 & 1 & 1 & 12 & 7 & 3 \\
\hline $\mathrm{C} 8$ & 7 & 7 & 8 & 0 & 0 & 0 \\
\hline
\end{tabular}

Table 5 Coefficients obtained by correlating the ranks of the crosses from multivariate probability predictions in the glasshouse $(\mathrm{GH})$ and at BB and MURR in the first clonal year with the number of clones that survived in the commercial breeding scheme at SCRI to the fourth, fifth and sixth clonal year trials

\begin{tabular}{llll}
\hline & \multicolumn{2}{l}{ Ranks of crosses based on multivariate probabilities } \\
& GH-1981 & BB-1982 & MURR-1982 \\
\hline Survived to 4th yr & -0.47 & -0.79 & -0.68 \\
Survived to 5th yr & -0.81 & -0.85 & -0.70 \\
Survived to 6th yr & -0.50 & -0.86 & -0.62 \\
\hline
\end{tabular}

progenies were not representative or selection carried out in the normal breeding scheme was not very effective before the third clonal year trial.

The cross (C7), which was ranked highest according to the multivariate probability prediction based on four variates, gives rise to 12 clones in the fourth year, 7 clones in the fifth year and 3 clones in the sixth year trials. Over all the crosses there is good agreement between the rank at the seedling or first clonal year stage and the number of clones that survived selection. Correlations between the rank of crosses at the seedling and first clonal year stage and the number of clones that survived commercial selection are shown in table 5. Only one correlation from the possible six between predicted ranking from the first clonal year and clones that survive was non-significant and this was only just outside the formal 5 per cent level. The correlations between predicted ranking estimated from seedlings were in general lower than those from the first clonal year trial. They were however clearly large enough in magnitude in that our earlier suggestion for their use would seem to be borne out in these late stages of this commercial breeding scheme.

\section{REFERENCES}

BROWN, J., CALIGARI, P. D. S., MACKAY, G. R. AND SWAN, G. E. L. 1984 The efficiency of seedling selection by visual preference in a potato breeding programme. J. Agric. Sci., 103, 339-346.

BROWN, J., CALIGARI, P. D. S., MACKAY, G. R. AND SWAN, G. E. L. 1987. The efficiency of visual selection in the early generations of a potato breeding programme. Ann. Appl. Biol., 110, 357-363.

CALIGARI, P. D. S. AND BROWN, J. 1986. The use of univariate cross prediction methods in the breeding of a clonally reproduced crop (Solanum tuberosum). Heredity, 57, 395401.

CALIGARI, P. D. S., BROWN, J. AND ABBOTT, R. J. 1986. Selection for yield and yield components in the early generations of a potato breeding programme. Theor. Appl. Genet., 73, 218-222.

CAligari, P. D. S., POWELl, W. AND JINKS, J. L. 1985. The use of doubled haploids in barley breeding. 2. An assessment of univariate cross prediction methods. Heredity, 53, 353358.

JINKS. J. L. AND POONI, H. S. 1976. Predicting the properties of recombinant lines derived by single seed descent. Heredity, 36, 253-266.

JINKS, J. L. AND POONI, H. S. 1980. Comparing predictions of mean performance and environmental sensitivity of recombinant inbred lines based upon $\mathrm{F}_{3}$ and triple test cross families. Heredity, 45, 305-312. 
KENDAL, M. S. 1962. Rank correlation methods, Griffin, London. pp. 91-106.

POONI, H. S. AND JINKS, J. L. 1978. Predicting the properties of recombinant imbred lines derived from single seed descent for two or more characters simultaneously. Heredity, 40, 349-361.

POWELL, W., CALIGARI, P. D. S., MCNICHOL, J. W., AND JINKS, J. L. 1985. The use of doubled haploids in barley breeding. 3 . An assessment of multivariate cross prediction methods. Heredity, 55, 249-254.

SCHERVISH, M. J. 1984. Multivariate normal probabilities with error bound. Appl. Stats, 33, 81-87.
SNAPE, J. W. 1982. Predicting the frequency of transgressive segregants for yield and yield components in wheat. Theor. Appl. Gent., 62, 127-134.

SNAPE, J. W. AND PARKER, P. 1986. Cross prediction in wheat using $\mathrm{F}_{\infty}$ data. In Proc. VIth meeting of Eucarpia section, Biometrics in Plant Breeding, Birmingham, U.K. p. 359-369.

THOMAS, W. T. B. TAPSELL, C. R. AND POWELL, W. 1986. The application of cross prediction in spring barley breeding. In Proc. VIth meeting of Eucarpia section, Biometrics in Plant Breeding, Birmingham, U.K. p. 80-89. 